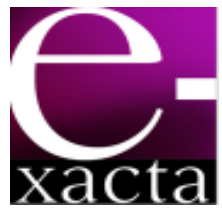

ISSN: 1984-3151

\title{
MINIMIZAÇÃO DO TERCEIRO HARMÔNICO ATRAVÉS DA LIGAÇÃO EM DELTA EM TRANSFORMADORES TRIFÁSICOS AO ATINGIR A SATURAÇÃO FERROMAGNÉTICA
}

\section{Minimizing of ThiRd HaRmonic By Delta ConNeCtion IN THREe- Phase Transformers to aChIEVE the FerRomagnetic SATURATION}

\author{
Maria Cecília Ferreira ${ }^{1}$; Marcus Vinícius Ferraz do Amaral ${ }^{2}$ \\ 1 Graduanda em Engenharia Elétrica. UniBH. Belo Horizonte, \\ MG, 2012. cecília.ferreira@hotmail.com \\ 2 Especialista em Engenharia Elétrica. PUC Minas, 1985 \\ Professor do Centro Universitário de Belo Horizonte - \\ UniBH. Belo Horizonte, MG. mvfamaral@gmail.com
}

Recebido em: 29/05/2012 - Aprovado em: 30/06/2012 - Disponibilizado em: 30/07/2012

\begin{abstract}
RESUMO: Neste trabalho é demonstrado que transformadores com núcleo ferromagnético, quando atingem o nível de saturação do seu núcleo, ficam propícios a componentes harmônicos, que em geral apresentam ondas com frequências maiores e múltiplas inteiras da fundamental - $60 \mathrm{~Hz}$, e distorcem a forma de onda de fluxo fundamental interferindo diretamente na qualidade de energia gerada. Para que um transformador obtenha um melhor desempenho é necessário que o mesmo trabalhe em níveis consideráveis de saturação do seu núcleo ferromagnético, a fim de obter o acoplamento desejado. Essa condição de operação gera essas distorções harmônicas indesejáveis. Baseado nos resultados obtidos durante o desenvolvimento desse trabalho, quando se utiliza a ligação em triângulo ou delta, em um dos enrolamentos do transformador, pode-se comprovar a minimização do terceiro harmônico, obtendo uma onda de tensão de linha menos distorcida, melhorando diretamente a qualidade de energia fornecida pelas concessionárias aos consumidores finais. Os dados também demonstraram que ao realizar o controle desses harmônicos de terceira ordem, através da ligação em triângulo no enrolamento primário do transformador, o mesmo é capaz de operar com condições de saturação do núcleo ferromagnético, sem oferecer uma onda de tensão distorcida, ou seja, o equipamento apresentará uma melhoria no desempenho, além de uma maior vida útil e menores perdas quando em atividade.
\end{abstract}

PALAVRAS-CHAVE: Transformadores. Saturação Magnética. Terceiro Harmônico. Ligação em Delta. Qualidade de Energia.

ABSTRACT: In this work it is shown that transformers with ferromagnetic core, when they reach the saturation level of its core, are prone to harmonic components that generally have larger frequencies and waves with multiple critical whole $-60 \mathrm{~Hz}$, and distort the fundamental flow waveform directly interfering in the quality of energy generated. For a transformer to get better performance, it is necessary that the same work at considerable levels of saturation of its ferromagnetic core, in order to obtain the desired coupling. This condition of operation generates these unwanted harmonic distortion. Based on the results obtained during the development of this work, when using the delta or Delta connection in one of the windings of the transformer can be supplied to minimization of third harmonic voltage wave getting a less distorted line, directly improving the quality of energy provided by the concessionaire to final consumers. The data also showed that when performing the track of these harmonics of the third order by Delta connection in the primary winding of the transformer, it is capable of operating with ferromagnetic core saturation conditions, without offering a distorted voltage waveform, i.e. the equipment shall submit an improvement in performance, as well as a longer life and lower losses while in activity. KEYWORDS: Transformers. Magnetic Saturation. Third Harmonic. Delta Connection. Power Quality. 


\section{INTRODUÇÃo}

Em todos os setores onde há aplicação de energia elétrica, tem-se um interesse de se obter qualidade de energia. Entre os fatores mais importantes para as máquinas elétricas obterem eficiência energética destacam-se: alto rendimento, baixa interferência eletromagnética, elevado fator de potência, baixo custo e maior vida útil do equipamento.

Os transformadores são máquinas estáticas conversoras de energia e são indispensáveis no campo da energia elétrica. Tais máquinas podem elevar e reduzir tensões com alto rendimento. Hoje em dia, com a elevação de tensão, pode-se transferir energia elétrica a imensas distâncias em escala industrial, de forma simplificada. É possível encontrar transformadores em sistemas elétricos de alta, média e baixa potência.

Como toda máquina elétrica, alguns fatores são importantes na análise de um transformador, são eles: fatores mecânicos como vibração e choques; fatores ambientais como temperatura e umidade; fatores elétricos como variação de tensão, frequência $e$ potência. Destacam-se entre fatores elétricos as interferências eletromagnéticas, em inglês EMI Eletromagnétic Interference. Pode-se descrever uma interferência eletromagnética como a ocorrência de alguma alteração no funcionamento de um determinado equipamento, quando o mesmo é exposto a campos eletromagnéticos. Em transformadores, essas interferências são causadas quando as correntes e tensões têm suas ondas distorcidas, devido ao aparecimento de componentes harmônicos. Essas interferências causam como resposta: perda no núcleo, redução da vida útil da máquina, aquecimento, os indesejáveis ruídos elétricos e, consequentemente, uma energia de baixa qualidade.

De acordo com Oliveira (1984), o primeiro fenômeno relacionado à presença de harmônicos foi observado quando linhas de transmissão eram colocadas próximas às linhas de comunicação e essas sofriam interferência das primeiras. Notou-se que algumas dessas linhas de transmissão apresentavam ondas com frequências maiores que a fundamental $-60 \mathrm{~Hz}$ e algumas eram até três vezes maior que a fundamental. Descobriu-se, então, que essa distorção nas ondas ocorria devido a componente de terceiro harmônico e de seus múltiplos impares, característica de um sistema trifásico de sequência zero, onde as correntes de terceiro harmônico estão em fase com as três linhas do sistema trifásico.

Para entender como ocorria essa interferência podese imaginar dois sistemas, um de transmissão e outro de comunicação, separados por uma distância consideravelmente grande. $\mathrm{O}$ campo magnético criado pelas ondas fundamentais será nulo devido a defasagem de $120^{\circ}$ entre os três campos. Já o campo produzido pelas ondas de frequência de $180 \mathrm{~Hz}$ será igual à soma dos campos de cada fase, ou seja, a amplitude será três vezes maior, já que elas estão em fase.

Notou-se que uma Força Eletromotriz - FEM - de terceiro harmônico era induzida na linha de comunicação, causando uma interferência no sistema. Essa interferência gerava ruídos nos receptores e tensões induzidas. Nos transformadores, além das consequências já citadas, notou-se também que algumas das tensões de fase eram maiores que o esperado, causando problemas de isolamento nos enrolamentos.

\section{RefERENCIAL TEÓRICO}

A saturação em transformadores de distribuição implica na baixa qualidade de energia fornecida pela concessionária aos consumidores. Essa energia de qualidade ruim pode gerar quedas de tensões elevadas e até a queima de equipamentos em 
residências. Em um sistema elétrico, equipamentos como transformadores, motores e geradores podem conter harmônicos. Um componente harmônico possui uma onda cuja frequência é múltipla da frequência fundamental e a amplitude é uma constante inteira da amplitude da onda fundamental.

O aparecimento de componentes harmônicos em transformadores ocorre pela relação não linear existente entre o fluxo magnético e a corrente de excitação.

\subsection{Circuitos Magnéticos e saturação do NÚCLEO}

De acordo com TORO (1999), todo condutor de comprimento $\ell$ quando submetido a um campo elétrico $\mathrm{E}$, cria em seu interior uma corrente elétrica i. Conforme a Lei de Ampere, um condutor percorrido por uma corrente elétrica, cria ao seu redor um campo magnético. Para aumentar tal campo poderia simplesmente submetê-lo a um nível elevado - acima do valor nominal - de corrente elétrica, o que não é aconselhável. Portanto, para obter um campo de maior intensidade, basta transformar o condutor em espiras. Sendo assim, quanto maior o número de espiras, maior a intensidade $\mathrm{H}$ do campo magnético. Essas espiras constituem os enrolamentos primário e secundário de um transformador. Tais enrolamentos comungam o mesmo núcleo e um fluxo magnetizante enlaça o primário e o secundário.

De acordo com Toro (1999), o núcleo de um transformador é constituído por materiais ferromagnéticos cuja permeabilidade relativa é muito maior que a do vácuo. Em um material ferromagnético desmagnetizado, seus domínios encontram-se desalinhados, ou seja, sem uma orientação correta. Ao submeter esse material a um campo magnético externo de intensidade $\mathrm{H}$, tem-se o alinhamento de seus domínios e pode-se dizer que o material encontra-se magnetizado. A densidade magnética $B$, é responsável pela orientação desses domínios e é diretamente proporcional à intensidade $\mathrm{H}$ vezes $\mu$, que é a permeabilidade do meio, conforme Eq. 1:

$$
B=\mu H\left[\frac{w b}{m^{2}}\right]
$$

onde: $B=$ Densidade Magnética ou Indução $\left[\mathrm{Wb} / \mathrm{m}^{2}\right]$, $\mu=$ Permeabilidade magnética [Wb/A.e.m] e $\mathrm{H}=$ Intensidade do Campo Magnético [A.e/m].

Segundo o mesmo autor, quando todos os domínios encontram-se alinhados, a energia fornecida para o alinhamento dos mesmos deixa de ser útil e a densidade $B$ deixa de crescer na mesma proporcionalidade de $\mathrm{H}$, ocorrendo então a saturação do material. Logo, conclui-se que B não pode ser tão elevado, para não saturar a máquina, nem tão baixo, para não interferir no acoplamento da máquina. A energia fornecida em excesso quando o núcleo está saturado distorce a onda de fluxo fundamental.

\subsection{O TRansformador de PotênCia}

Toro (1999) afirma que um transformador é formado por um núcleo de material ferromagnético, em torno do qual estão enroladas duas bobinas. O fluxo magnético criado pela corrente elétrica envolve as duas bobinas em torno do núcleo.

Para o mesmo autor, o funcionamento de um transformador baseia-se nas leis do eletromagnetismo criadas por Faraday e Lenz. Um campo magnético variável que circula em volta de um condutor faz surgir nele uma tensão induzida variável. O núcleo do transformador é comum às bobinas de primário e secundário. Quando se alimenta umas dessas bobinas com uma tensão nominal, tem-se um fluxo magnético envolvendo esse núcleo. Sabe-se que o transformador é uma máquina de corrente alternada, logo, o fluxo magnético produzido é variante no tempo, já que a corrente alternada oscila em $60 \mathrm{~Hz}$. Se o primário é 
alimentado, o fluxo ao percorrer o secundário (lado que se conecta a carga) provoca o aparecimento de uma tensão alternada na bobina de secundário por indução magnética. Essa tensão é chamada de tensão induzida que é proporcional ao número de espiras da bobina e depende da variação do fluxo. Essa tensão induzida é demonstrada pela Eq. 2 que comprova a Lei de Lenz.

$$
V_{i n d}=-N \frac{\partial \phi}{\partial t}[V]
$$

onde: $V_{\text {rd }}=$ Tensão Induzida [V], $N=$ Número de Espiras e $\phi=$ Fluxo Magnético [Wb].

\subsection{CuRVA DE MAGNETIZAÇÃo dOS MATERIAIS} FERROMAGNÉTICOS QUE COMPÕEM O NÚCLEO DO TRANSFORMADOR

Segundo GUEDES (1992) o núcleo de um transformador é construído com materiais ferromagnéticos que atingem uma alta magnetização quando são submetidos a um campo magnético externo. Sabendo que a densidade $B$ e a intensidade $\mathrm{H}$ são proporcionais, quando o material é inicialmente magnetizado, B assume valores crescentes relacionados a $\mathrm{H}$, formando uma curva de magnetização. Logo, a curva de magnetização dos transformadores é gerada a partir da densidade de fluxo $\mathrm{B}$ versus a intensidade de fluxo $\mathrm{H}$, como mostra a FIG.1. Ela varia para cada transformador de corrente, devido a não-linearidade magnética dos materiais que constituem os núcleos.

De acordo com o mesmo autor, os ciclos de magnetização passam a ser subsequentes após a primeira magnetização, onde, um ciclo depende do anterior. Sendo assim, a magnetização passa a ser simétrica e alternada entre os valores de indução magnética, criando um ciclo fechado $\mathrm{B}-\mathrm{H}$, chamado ciclo ou laço de histerese, como mostra a FIG. 2.

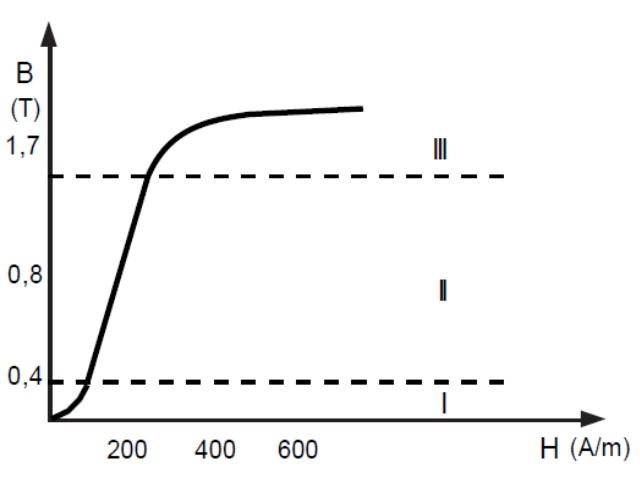

Figura 1 - Curva de magnetização Fonte - GUEDES, 1992, p.2.

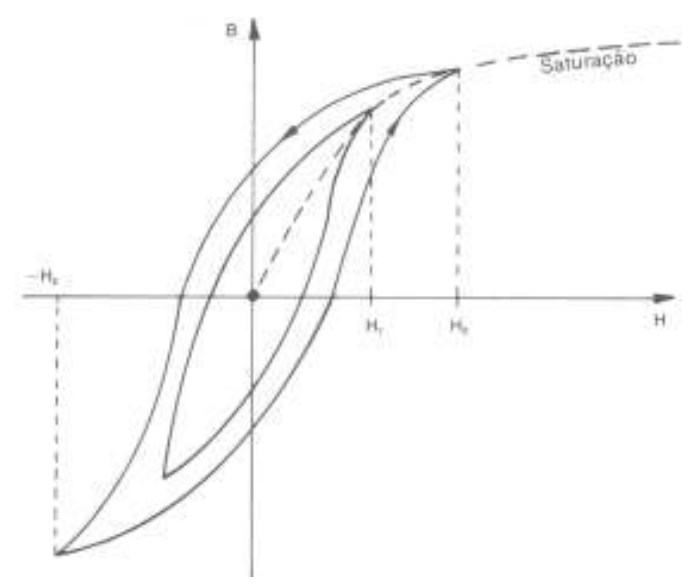

Figura 2 - Laço de Histerese Fonte - GUEDES, 1992, p.2.

\subsection{Geração de haRmônicos Ímpares em TRANSFORMADORES TRIFÁSICOS}

Os circuitos magnéticos dos transformadores são excitados por fontes CA e não CC. Quando os transformadores operam em regime permanente, pode-se considerar que a indução é constante, ou seja, o fluxo não é diretamente proporcional à corrente, por isso a linearidade é desprezível.

De acordo com TORO (1999), existem duas análises a serem feitas para compreender a geração de harmônicos. Considerando os efeitos da histerese 
(curva B-H): seja um transformador convencional onde a tensão aplicada no primário tenha a forma de onda senoidal. Consequentemente somente uma corrente de magnetização senoidal é fornecida ao primário.

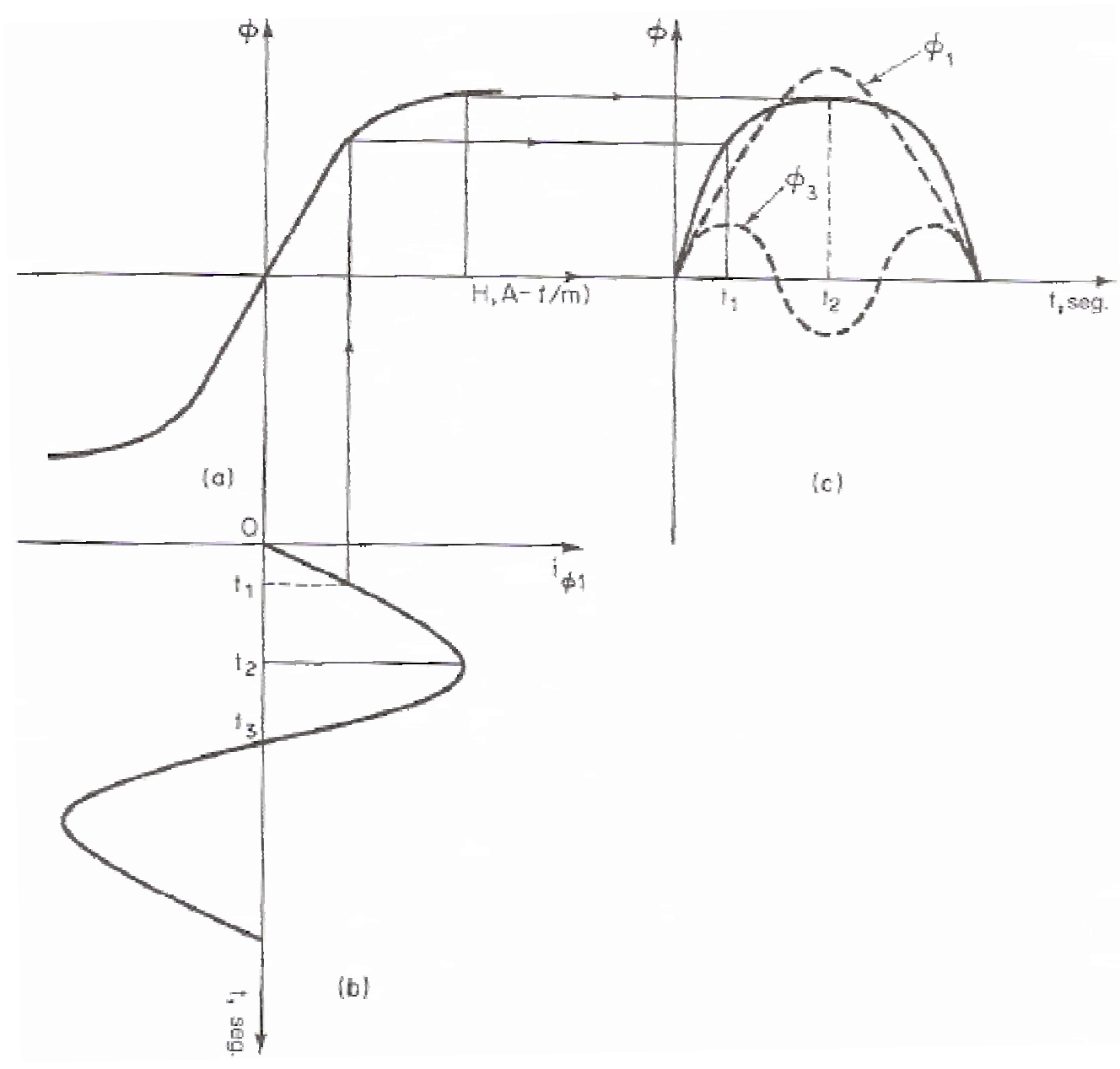

Figura 3 - Efeito de uma corrente de magnetização senoidal. (a) curva de magnetização mostrando saturação sob forças de magnetização elevadas e sem histerese; (b) corrente de magnetização senoidal suposta como circulando da fonte de tensão para a bobina de primário; (c) a onda de fluxo resultante com o topo abaulado produzida pela corrente de (b).

Fonte - TORO, 1999, p.55.

O mesmo autor afirma que, quando se projeta a corrente de magnetização sobre a curva de magnetização, uma onda de fluxo com topo abaulado como mostra a FIG. 3(c), é produzida. Essa onda possui duas componentes de harmônicos: uma fundamental $\Phi 1$ e uma negativa de $3^{\mathrm{a}}$ ordem, $\Phi 3$. $\Phi 1$ induzirá uma tensão E1 na bobina de primário que é igual, mas oposta a tensão que alimenta a bonina de primário, V1. Da mesma forma, Ф3 induzirá uma tensão E3 no primário, mas esta, por sua vez é uma senóide, por isso, não haverá outra componente igual e oposta a ela na fonte de tensão do primário. Sendo assim a tensão induzida de terceiro harmônico fica livre e produz uma corrente de terceiro harmônico negativa. Esta, por sua vez, se soma à componente fundamental positiva, de tal forma que a corrente de 
magnetização seja o resultado da superposição das duas componentes (FIG. 4).

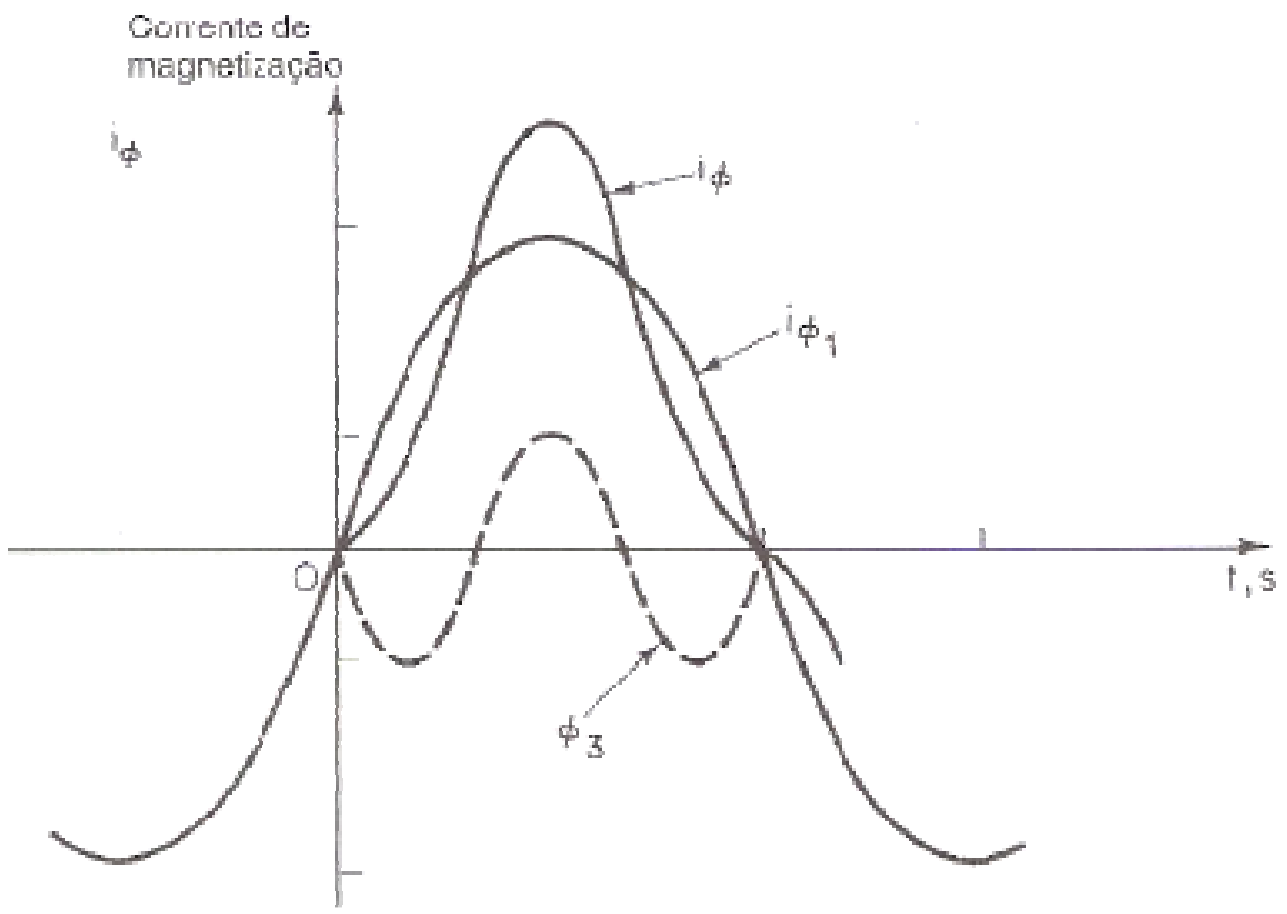

Figura 4 - Forma de onda da corrente de magnetização do Transformador desprezando a histerese.

Fonte - TORO, 1999, p.56.

De acordo com GUEDES (1992), é importante identificar as consequências da existência do terceiro harmônico nos transformadores trifásicos de potência, onde a condição de circulação desse harmônico condiciona a escolha do tipo de ligação das bobinas dos enrolamentos do transformador, devido aos problemas criados em redes de telecomunicações pela circulação do terceiro harmônico nas linhas de transporte de energia.

Segundo LEÃO (2010) o aparecimento do terceiro harmônico gera distorções na forma de onda das tensões e correntes. Quando um transformador opera com uma densidade maior, ele exige um material com menor nível de magnetização. Conclui-se então que a corrente de magnetização irá conter além da componente fundamental todos os componentes harmônicos ímpares, sendo que desses o terceiro harmônico é o mais significativo por duas razões: pela sua amplitude, que é um terço da fundamental e pela sua frequência, que é o triplo da fundamental. Porém, o quinto harmônico também possui sua parcela de significância, já que sua amplitude é um quinto da fundamental e sua frequência é o quíntuplo da fundamental.

Harmônicos na onda de tensão aumentam as perdas no ferro, enquanto harmônicos na onda da corrente aumentam as perdas no cobre. Ambas as perdas variam com a frequência, logo, quanto maior a frequência, maior a perda. Se as perdas são elevadas, o aquecimento da máquina também se eleva e a vida útil do equipamento é reduzida devido à degradação do material isolante no interior do transformador.

\subsection{TEOREMA DAS COMPONENTES SIMÉTRICAS}

De acordo com STEVENSON (1986), no ano de 1918, Fortescue apresentou uma ferramenta para análise de 
circuitos polifásicos desequilibrados. Desde então o método de Fortescue foi abordado em diversas pesquisas experimentais. Esse método estuda as faltas assimétricas em sistemas de transmissão que podem ser: curto-circuito, impedâncias de linhas para a terra ou impedância entre linhas e condutores em aberto.

Segundo STEVENSON (1986), o teorema das componentes simétricas demonstra que um sistema desequilibrado de $\mathrm{n}$ fasores pode ser decomposto em outro sistema de $\mathrm{n}$ fasores equilibrados que são os componentes simétricos dos originais. Ambos são idênticos no comprimento e os seus ângulos com fasores adjacentes também são iguais.

Tomando como base um sistema trifásico desequilibrado, através do teorema das componentes simétricas, pode-se decompor esse sistema em um conjunto de 3 sistemas equilibrados de fasores:

- Componentes de sequência positiva: Composto por 3 fasores iguais em módulo, defasados de $120^{\circ}$ entre si que possuem a mesma sequência dos fasores originais.

- Componentes de sequência negativa: Composto por 3 fasores iguais em módulo defasados de $120^{\circ}$ entre si, mas com a sequência inversa dos fasores originais.

- Componentes de sequência zero: Composto por 3 fasores iguais em módulo com defasagem nula entre si.

Denominando a, b e c como as três fases do sistema têm-se uma sequência positiva de fases abc das tensões e das correntes e uma sequência negativa de fases acb das tensões e das correntes. Os fasores originais representam as tensões designados por $\mathrm{Va}$, $\mathrm{Vb}$ e $\mathrm{Vc}$ e os índices 1, 2 e 0 representam os componentes de sequência positiva, negativa e zero, respectivamente. Assim, os fasores desequilibrados originais representam a somas das componentes de sequência positiva, negativa e zero como mostra a FIG.5.

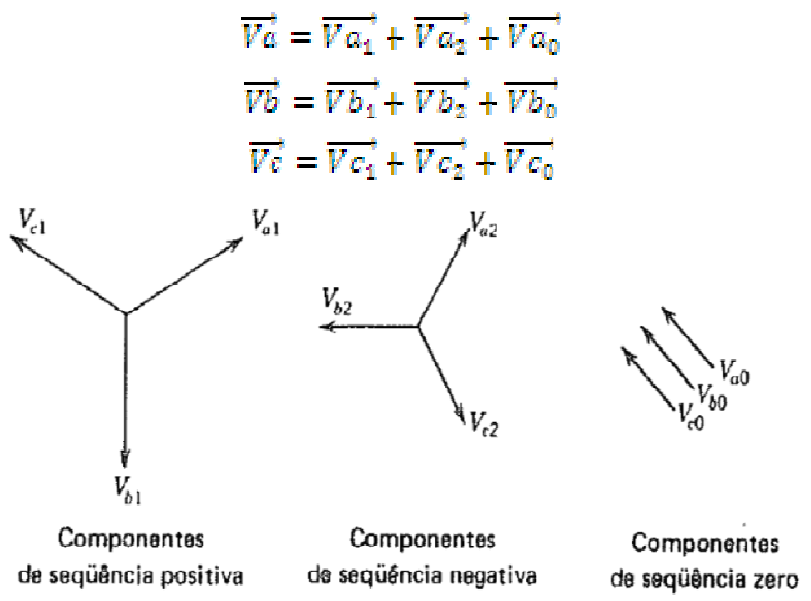

Figura 5 - Conjunto de fasores equilibrados que são componentes de fasores desequilibrados de um sistema trifásico.

Fonte - STEVENSON, 1986, p. 296.

\subsubsection{LIGAÇÃo EM ESTRELA (Y)}

Segundo MICHELS (s/data), em um sistema trifásico alimentado por uma carga desequilibrada e conectado em estrela (FIG. 6), o desequilíbrio da carga reflete na ligação em estrela causando deslocamento de neutro, pois, haverá regulações de tensão diferentes entre as fases. A ligação em estrela apresenta distorções consideráveis nas tensões causadas pelo terceiro harmônico que é gerado pelas características magnetizantes do núcleo do transformador.

De acordo com o mesmo autor, transformadores trifásicos operam com defasagem das correntes de $120^{\circ}$ entre si, portanto, os componentes harmônicos de sequência positiva e negativa se anulam devido à simetria do sistema. Já os componentes harmônicos de sequência zero estão em fase com as correntes e se somam a elas, por isso geram distorções na forma de onda. Pode-se solucionar esse problema utilizando a ligação em delta ou triângulo. 


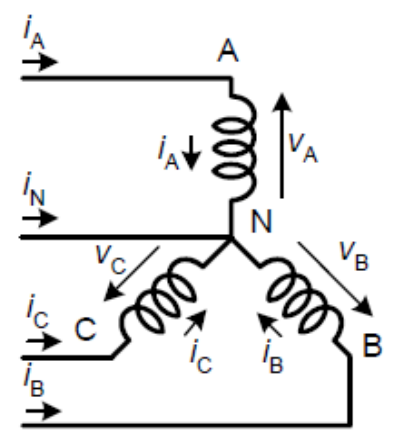

Figura 6 - Representação da Ligação em estrela.

Fonte - MICHELS, s/data, p. 3.

\subsubsection{Redes de SequênCia Zero e Ligação EM TRIÂNGULO (D)}

Stevenson (1986) afirma que num sistema trifásico, as correntes de sequência zero são iguais em módulo nas três fases do sistema. Portanto, só existe circulação dessas correntes se o caminho for fechado, característica de uma ligação em triângulo (FIG. 7).

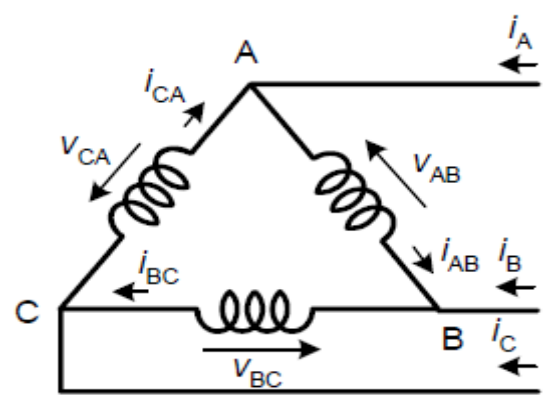

Figura 7 - Representação da Ligação em Delta ou Triângulo.

Fonte - MICHELS, s/data, p. 3.

Segundo Stevenson (1986), a ligação em triângulo tem como finalidade criar um caminho sem retorno, ou seja, um circuito fechado para a circulação das correntes harmônicas de sequência zero. Além disso, outra utilidade importante é fornecer um caminho fechado para as correntes de falta do sistema. Como o circuito é fechado, não há necessidade de criar um ponto de aterramento para o sistema, já que não existe um ponto comum entre as fases.
Em um transformador trifásico, as tensões de fase são três ondas iguais, simétricas e defasadas de $120^{\circ}$ entre si. Cada uma dessas tensões das fases A, B e $C$, possui uma onda de terceiro harmônico em fase com a onda de tensão, de amplitude um terço menor e de frequência três vezes maior que a fundamental como pode ser observado no gráfico da FIG. 8.

- A forma de onda em azul representa a tensão da fase $A$;

- A forma de onda em verde representa a tensão da fase $B$;

- A forma de onda em vermelho representa a tensão da fase C;

- A forma de onda em preto representa a componente de terceiro harmônico.

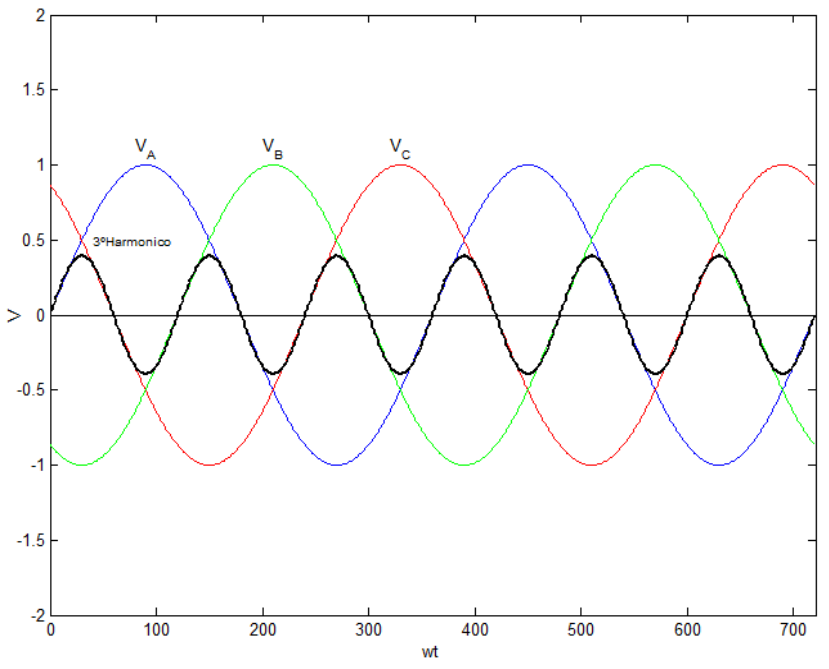

Figura 8 - Tensões de Fase $\mathrm{Va}, \mathrm{Vb}$ e $\mathrm{Vc}$ de um Sistema Trifásico e a onda de Terceiro Harmônico.

As componentes de sequência zero possuem a mesma fase, direção e sentido, ou seja, são vetores iguais como mostra a FIG. 9. Por isso, pode-se dizer que o terceiro harmônico é igual a componente de sequência zero. 


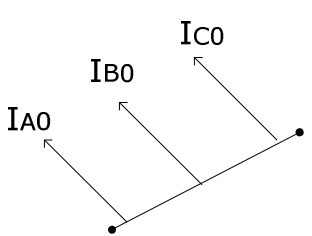

Figura 9 - Componentes de Sequência Zero.

Na ligação em Triângulo as correntes que circulam no caminho fechado são representadas na FIG. 10.

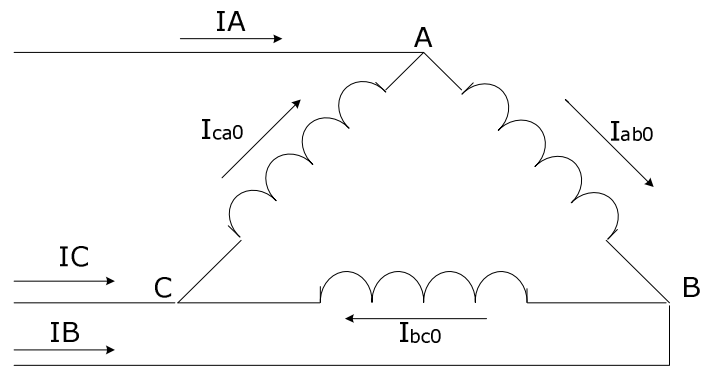

Figura 10 - Ligação em Triângulo.

Pela Lei de Kirchhoff das Correntes (LKC), sabe-se que a somatória das correntes incidentes em um nó de um circuito elétrico é sempre nula. Utilizando o nó A da FIG. 10 como referência, tem-se:

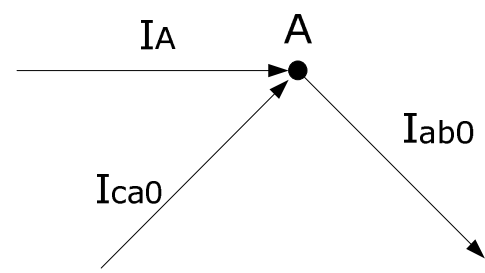

Figura 11 - Correntes Incidentes no nó A.

Onde,

$$
I_{A}+I_{c a v}=I_{a b D}
$$

Portanto,

$$
I_{A}=I_{a b 0}-I_{e a 0}
$$

Como $I_{a b 0}=I_{a a 0}$, as duas se cancelam, logo:$$
I_{A}=0
$$

Sendo assim, não existe corrente de terceiro harmônico na corrente de linha.
\end{abstract}

\section{Metodologia}

De acordo com GIL (2010), essa é uma pesquisa experimental que consiste em estudar profundamente um objeto, de forma que permita seu amplo e detalhado conhecimento. Essa pesquisa aconteceu através do aprimoramento de ideias e da utilização de técnicas de coletas de dados para solucionar o problema. As informações contidas em livros, revistas científicas, teses, relatórios científicos, cuja autoria é conhecida, foram propícios para o enfoque do problema proposto. Já a coleta de dados foi feita através dos ensaios realizados em laboratório, onde a análise de resultados possibilitou conclusões para solucionar o problema. Os procedimentos metodológicos dividem-se em cinco etapas de pesquisa:

A primeira etapa consistiu na revisão bibliográfica, cuja finalidade era propor um embasamento teórico e potencializar o conhecimento do pesquisador para discussão dos resultados sobre o tema proposto. A pesquisa sobre ação do campo magnético em núcleos ferromagnéticos foi feita a partir da leitura de bibliografias sobre o assunto, bem como teses e artigos disponíveis na internet.

$\mathrm{Na}$ segunda parte foi feita uma abordagem da formação de componentes harmônicos e o estudo de seus efeitos em transformadores, onde também se utilizou livros e sites de pesquisa como auxílio.

Já na terceira parte, foram identificadas as falhas consequentes do aparecimento dos harmônicos além das perdas de eficiência dos transformadores devido às interferências causadas. Isso foi feito através da abordagem do assunto em materiais encontrados no acervo da biblioteca, além de várias experiências em laboratório que comprovaram todo o estudo realizado. 
Na quarta parte da pesquisa, foi proposta uma solução para a redução do terceiro harmônico, através da ligação em triângulo no enrolamento primário do transformador. A solução proposta foi satisfatória através dos resultados obtidos no laboratório do UniBH, onde foi possível observar uma onda menos distorcida na ligação triângulo-estrela. Ou seja, essa medida irá minimizar perdas e oferecer uma energia "mais limpa". Através do estudo experimental e da análise dos resultados, foi possível alcançar o objetivo principal desse trabalho: reduzir os efeitos das componentes de terceiro harmônico nos transformadores.

\section{Resultados e Discussões}

Para a obtenção de resultados que comprovem a solução proposta neste trabalho, foram realizados alguns ensaios em transformadores no laboratório. Durante as experiências feitas, foram coletados dados de medição além de fotos que retratam o que foi analisado.

\subsection{ANÁLISE EXPERIMENTAL I: CURVA BXH COM NÚCLEO FERROMAGNÉTICO SATURADO}

O núcleo de um transformador constituído de material ferromagnético possui características não lineares. Sabe-se que cargas não lineares são dispositivos saturáveis que produzem correntes distorcidas mesmo quando alimentadas por uma fonte não distorcida (senoidal). A saturação desse núcleo ocorre quando o a indução magnética deixa de crescer na mesma proporcionalidade que a intensidade magnética, de tal modo que o campo magnético gerado se torna limitado. Essa relação entre as constantes $\mathrm{B}$ e $\mathrm{H}$ pode ser expressa pela permeabilidade magnética $\mu$, que representa um acoplamento desejável para o transformador operar. A curva de magnetização demonstrada na FIG. 1 representa essa relação.
O quadro de Epstein é um aparelho muito utilizado para mensurar perdas magnéticas. Nesse trabalho, ele foi utilizado para comprovar que em condições normais o núcleo ferromagnético atinge o nível máximo de saturação ficando sujeito a distorções harmônicas. A FIG. 12 demonstra o quadro de Epstein.

De acordo com a NBR 5161 (1977), o quadro de Epstein é indicado como referência para caracterização magnética de aço laminado. O Epstein é um dispositivo eletromagnético constituído por dois enrolamentos, em que o primário é responsável pela geração do campo eletromagnético e, o secundário, pelo fluxo magnético que é induzido. No quadro utilizado nessa análise experimental, têm-se quatro bobinas de $\mathrm{N}=600$ espiras constituídas de condutor de comprimento $\ell=2 \mathrm{~m}$, onde as bobinas são ligadas em série. A seção reta do condutor é $S=13,4 \mathrm{~cm}^{2}=$ $0.00134 \mathrm{~m}^{2}$. A FIG. 13 mostra o quadro de Epstein utilizado no experimento realizado no Laboratório de Máquinas Elétricas na PUC Minas.

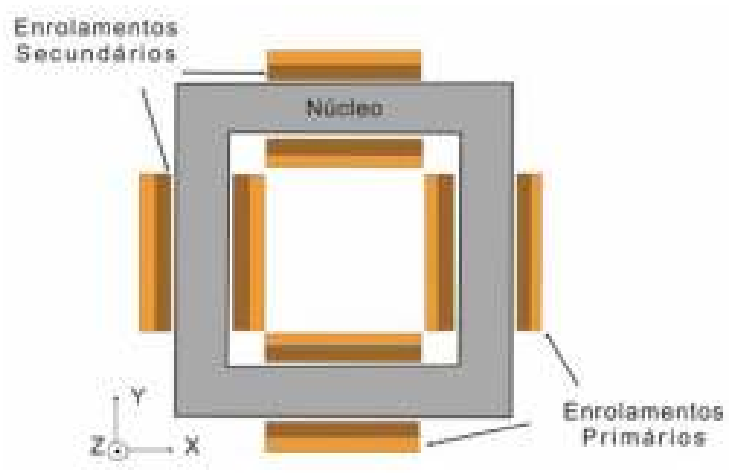

Figura 12 - Representação do quadro de Epstein Fonte - SILVA JÚNIOR, 2007, p. 9. 


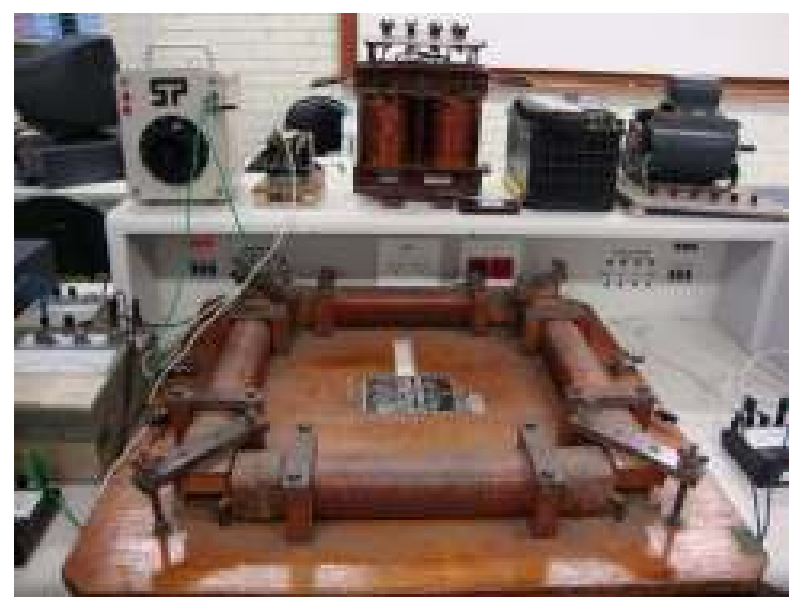

Figura 13 - Detalhe do Quadro de Epstein utilizado no experimento no laboratório de Máquinas Elétricas da PUC Minas.

O teste no Epstein foi realizado a partir do diagrama da FIG. 14 que representa um transformador, uma fonte de tensão alimentando o enrolamento primário e os aparelhos de medições conectados.

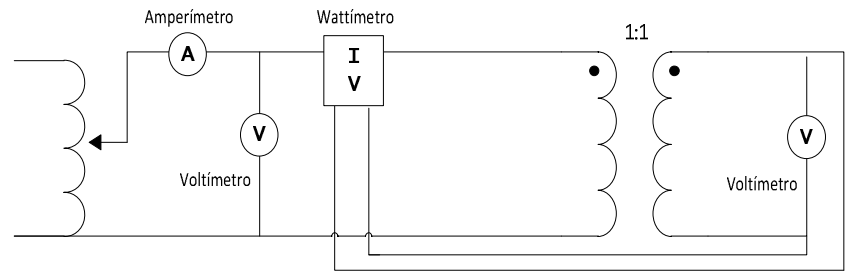

Figura 14 - Representação da montagem feita no laboratório de Máquinas Elétricas na PUC Minas.

O ensaio feito no laboratório consistiu em traçar a curva de magnetização com frequência de $60 \mathrm{~Hz}$. Foram estipulados valores de tensão a serem aplicados no enrolamento primário do Epstein $\left(V_{\text {RMSentrada }}\right)$ e as medições de tensão, corrente e potência nos dois enrolamentos do Epstein encontram-se na TAB. 1.
Tabela 1

Valores Medidos nos Enrolamentos do Epstein

\begin{tabular}{c|c|c|c|c}
\hline \multirow{2}{*}{$V_{\text {RMSentrada }}$} & \multicolumn{4}{|c}{ Valores medidos } \\
\cline { 2 - 5 } & $\mathrm{I}[\mathrm{A}]$ & $\mathrm{V}_{\text {RMS1 }}[\mathrm{V}]$ & $\mathrm{V}_{\text {RMS2 }}[\mathrm{V}]$ & $\mathrm{P}[\mathrm{VA}]$ \\
\hline 92 & 0.1 & 92 & 92 & 6.4 \\
\hline 120 & 0.2 & 120 & 120 & 12,0 \\
\hline 142 & 0.4 & 142 & 142 & 17,0 \\
\hline 155 & 0.6 & 155 & 155 & 18,0 \\
\hline 165 & 0.8 & 165 & 165 & 20,5 \\
\hline 172 & 1.0 & 172 & 172 & 23,6 \\
\hline 179 & 1.5 & 179 & 179 & 27,8 \\
\hline 186 & 2.0 & 186 & 186 & 29,0 \\
\hline 192 & 2.5 & 192 & 192 & 32,0 \\
\hline 194 & 3.0 & 194 & 194 & 34,0 \\
\hline
\end{tabular}

A partir dos dados obtidos nas medições pode-se fazer um do estudo eletromagnético a fim de calcular os valores de $\mathrm{B}$ e $\mathrm{H}$ e obter a curva de magnetização.

A Intensidade de campo magnético $\mathrm{H}$ pode ser obtida a partir do número de espiras $\mathrm{N}$ multiplicado pela corrente I dividido pelo comprimento $\ell$, conforme Eq. 5 :

$$
H=\frac{N_{H} I}{l}\left[A \cdot \frac{\nu}{m}\right]
$$

Onde: $\mathrm{H}$ é a Intensidade do campo [A.e/m], $\mathrm{N}$ é o números de espiras da bobina, I a corrente $[\mathrm{A}]$ e $\ell$ o comprimento do condutor [m].

Já a Densidade de campo magnético $B$, ou Indução magnética é obtida através da Eq. 1, vista na seção 2.1 .

Sabe-se que quanto menor a bitola do condutor de seção $S$ no núcleo, mais difícil se torna a passagem de linhas de campo magnético. Portanto, pode-se obter o fluxo magnético máximo a partir da Eq. 6:

$$
\psi_{\max }-B . S[W \mathrm{~W}]
$$


Onde: $\phi_{\max }$ representa o fluxo magnético [Wb], B a Indução magnética $\left[\mathrm{Wb} / \mathrm{m}^{2}\right]$ e $\mathrm{S}$ a seção reta do condutor $\left[\mathrm{m}^{2}\right]$.

O fluxo máximo também pode ser obtido através do produto entre a Indução Magnética, a área do condutor o cosseno do ângulo formado, ou seja:

$$
\phi_{\max }=3 \cdot S \cdot \cos (\omega t)[W b](7)
$$

Portanto, o fluxo total pode ser reapresentado a partir da Eq. 7.

$$
\phi=\phi_{\max } \cdot \cos (\omega t)[w b]
$$

Como já foi dito, um transformador é uma máquina de corrente alternada, onde o fluxo magnético varia no tempo já que a frequência oscila em $60 \mathrm{~Hz}$. Quando se alimentou o enrolamento primário do Epstein, um fluxo percorreu o enrolamento secundário, gerando uma tensão alternada na bobina de secundário por indução magnética. Essa tensão induzida é proporcional ao número de espiras da bobina, e pode ser demonstrada a partir a Eq. 2, dada na seção 2.2, que comprova a Lei de Lenz.

Como o fluxo é variante no tempo, pode-se substituir (8) em (2) para obter a tensão induzida em relação ao fluxo magnetizante.

$$
\begin{aligned}
& V_{i n d}=\frac{-N\left(d \phi_{\max } \cos (\omega t)\right)}{d t} \\
& \int V_{\text {ind }}=\int \frac{-N d \phi_{\max } \cos (\omega t)}{d t} \\
& V_{\text {ind }}-N \omega \phi_{\max } \cdot \operatorname{sen}(\omega t)
\end{aligned}
$$

Como $\omega=2 \pi f[I I z]$, então:

$$
V_{\text {imd }}=-2 \pi \cdot f \cdot N \cdot \phi_{m a x} \cdot \operatorname{sen}(\omega t)
$$

$$
V_{\text {of }}=\frac{2 \pi}{\sqrt{2}} \cdot f \cdot N \cdot \phi \text { onde } \phi=B . S
$$

$$
\begin{aligned}
& V_{e f}=4,44 . f \cdot N \cdot B \cdot S \\
& B=\frac{V_{g f}}{4,44 . f \cdot N \cdot S}
\end{aligned}
$$

Substituindo os valores na Eq. 10 tem-se:

$$
B=\frac{V_{x /}}{4,44.60,600,0,00134}\left[\mathrm{~Wb} / \mathrm{m}^{2}\right]
$$

Substituindo os valores na Eq. 5 tem-se:

$$
H=\frac{600 . I}{2}\left[A \cdot \frac{e}{m}\right]
$$

Através dos dados obtidos nas medições (TAB.1) foi possível calcular os valores de $\mathrm{B}$ e $\mathrm{H}$ representados na TAB. 2 e gerar o gráfico da curva de saturação que é mostrado na FIG. 15.

Tabela 2

Valores de $\mathrm{B}$ e $\mathrm{H}$ encontrados

\begin{tabular}{c|c}
\hline \multicolumn{2}{c}{ Valores Calculados } \\
\hline$H(X)$ & $B(Y)$ \\
\hline 0 & 0 \\
\hline 30,00 & 0,430 \\
\hline 60,00 & 0,560 \\
\hline 120,00 & 0,663 \\
\hline 180,00 & 0,724 \\
\hline 240,00 & 0,770 \\
\hline 300,00 & 0,803 \\
\hline 450,00 & 0,836 \\
\hline 600,00 & 0,868 \\
\hline 750,00 & 0,896 \\
\hline 900,00 & 0,906 \\
\hline
\end{tabular}

Sendo assim, 


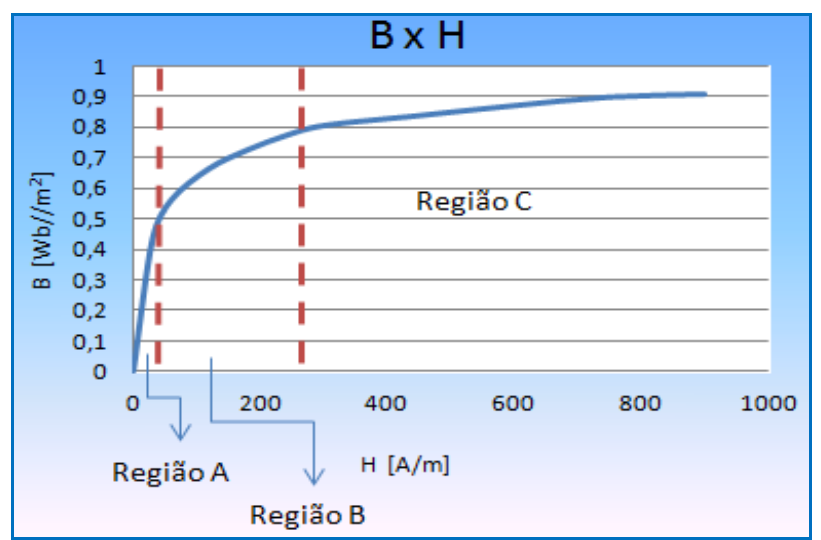

Figura 15 - Gráfico da Curva de Saturação Magnética $\mathrm{BxH}$

\subsubsection{DISCUSSÃO DA ANÁLISE EXPERIMENTAL I}

O núcleo do Toróide de Epstein, constituído de material ferromagnético, até a energização do equipamento encontrava-se desmagnetizado, ou seja, seus domínios estavam desalinhados. Ao submetê-lo a um campo magnético externo de intensidade $H$, seus domínios tenderam a se organizar seguindo a mesma orientação da densidade magnética B. A medida que o campo magnético foi sendo aumentado, houve uma maior dificuldade em se obter novos alinhamentos. Quando todos os domínios encontraram-se alinhados, a energia fornecida para o alinhamento dos mesmos deixou de ser útil e a densidade $B$ deixou de crescer na mesma proporcionalidade de $\mathrm{H}$, ocorrendo a saturação do material.

Assim, é possível identificar as três regiões da curva de saturação:

- Região A: Região onde a permeabilidade do meio $(\mu)$ é constante;

- Região B: "Joelho" da curva. Região mais adequada para a operação de máquinas elétricas. O maior valor que $B$ pode assumir antes da saturação;
- Região C: Região de saturação onde o aumento de $\mathrm{H}$ não influencia mais em $\mathrm{B}$.

\subsection{ANÁLISE EXPERIMENTAL II: LIGAÇÃo Y-Y E D-Y}

Em um transformador o fluxo magnético que é gerado no núcleo de ferro pelo enrolamento primário atinge o enrolamento secundário acoplando os dois enrolamentos. Para que ocorra esse acoplamento, é necessário que o equipamento atinja um nível de saturação, de tal forma que, a permeabilidade magnética do material altamente condutor que compõe o núcleo, garanta esse enlace.

A curva BxH da FIG. 15 demonstra a necessidade do transformador trabalhar em um nível de densidade de fluxo a fim de obter acoplamento entre as bobinas. Esse nível de $B$ leva a máquina à saturação magnética, causando distorção na amplitude das ondas de tensão de fase e tensão de linha da saída do transformador. Esse fenômeno foi observado durante o experimento realizado no Laboratório de Eletrônica do UniBH.

Durante o experimento foi utilizado:

- Dois Transformadores KING de 1 KVA, $240 \mathrm{~V}$ (Triângulo)/ 440 V(Estrela);

- Osciloscópio TECTRONIX TDS210 60 Hz/ (1GS/s) com dois canais $(\mathrm{CH} 1$ e $\mathrm{CH} 2)$;

- Fonte de Alimentação Trifásica $220 \mathrm{~V}$.

O transformador utilizado possui seu enrolamento secundário ligado em estrela, por isso utilizou-se dois transformadores iguais, modificando apenas a ligação do enrolamento primário: no primeiro transformador ligou-se o primário em Estrela $(\mathrm{Y}-\mathrm{Y})$ e no segundo, ligou-se o primário em Triângulo (D-Y). A FIG. 16 mostra a montagem feita. 


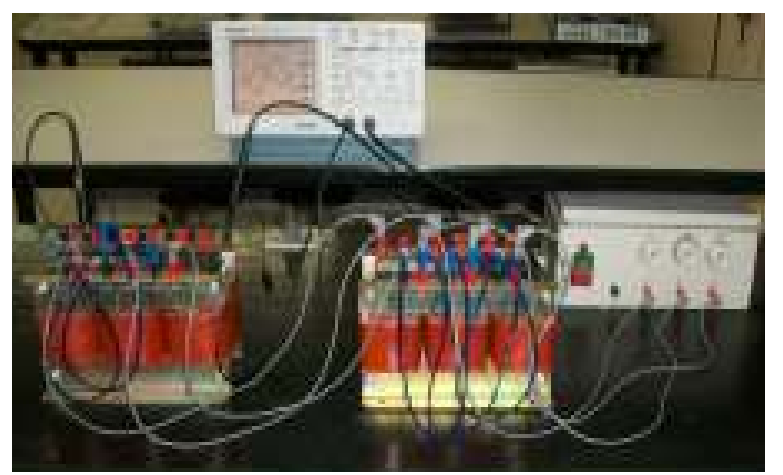

Figura 16 - Imagem da Montagem no Laboratório para a segunda análise experimental.

A ligação dos primários em Estrela e em Triângulo respectivamente foi feita a partir da instrução contida no próprio equipamento (FIG. 17).

\begin{tabular}{|l|l|}
\hline \multicolumn{2}{|c|}{ Entrada de energia } \\
\hline 1 Ligacao delta $(220 \mathrm{~V})$ & 2 Ligacao estrela $(380 \mathrm{~V})$ \\
\hline Fechar $\times 2 \operatorname{com} \times 3$ & \\
\hline Fechar $\times 4 \operatorname{com} \times 5$ & Fechar $\times 1 \operatorname{com} \times 3 \operatorname{com} \times 5$ \\
\hline Fechar $\times 6 \operatorname{com} \times 1$ & \\
\hline Ligar $220 \mathrm{~V}$ em $\times 2, \times 4, \times 6$ & Ligar $380 \mathrm{~V}$ em $\times 2, \times 4, \times 6$ \\
\hline
\end{tabular}

Figura 17 - Imagem da Instrução para ligação do Enrolamento Primário.

Em seguida alimentou-se o transformador ligado em D-Y com $220 \mathrm{~V}$ a partir da fonte de alimentação trifásica. Para alimentar o transformador ligado em Y-Y foi necessário $380 \mathrm{~V}$. Para isso, utilizou-se a saída do transformador D-Y de $380 \mathrm{~V}$.

As FIGS. 18 e 19 mostram os detalhes das ligações de cada transformador.

Através dos canais $\mathrm{CH} 1$ e $\mathrm{CH} 2$ do osciloscópio foi possível observar as formas de onda das tensões de fase e de linha de cada transformador e a partir daí fazer as comparações necessárias.

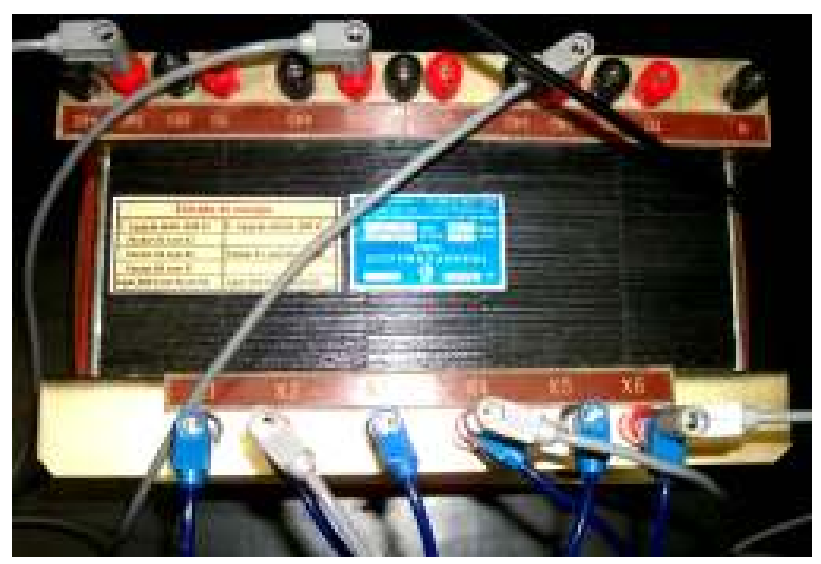

Figura 18 - Imagem da conexão D-Y (Delta-Estrela) no transformador alimentado com $220 \mathrm{~V}$.

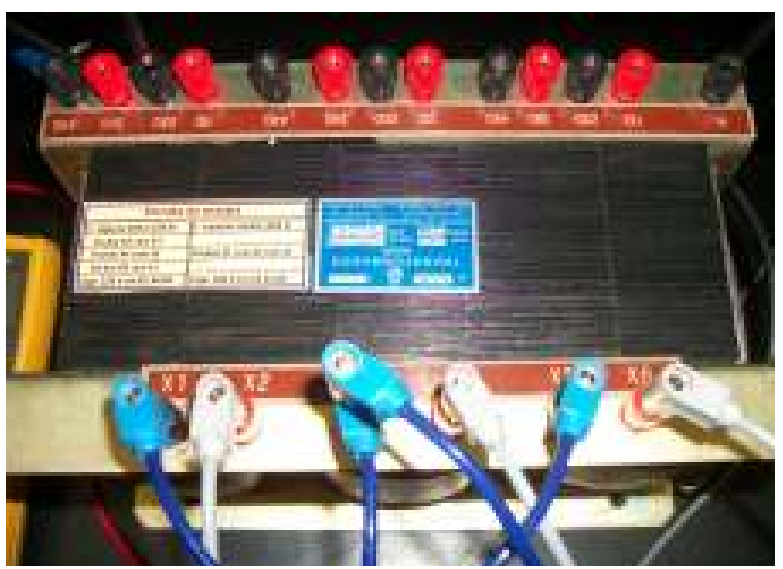

Figura 19 - Imagem da conexão Y-Y (Estrela-Estrela) no transformador alimentado com $380 \mathrm{~V}$.

Na FIG. 20, estão demonstradas as formas de onda da tensão de fase e da tensão de linha, do transformador ligado em D-Y. Já na FIG. 21, estão demonstradas as formas de onda da tensão de fase e da tensão de linha, do transformador ligado em $Y$-Y.

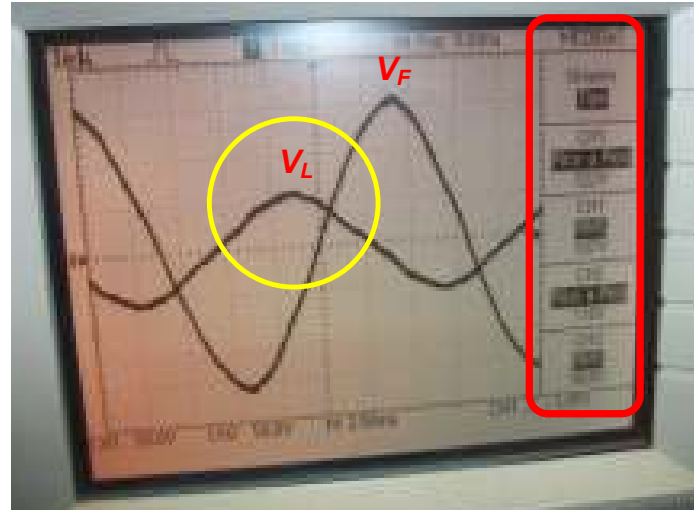

Figura 20 - Formas de Onda de $V_{F}$ e $V_{L}$ do transformador conectado em D-Y. 


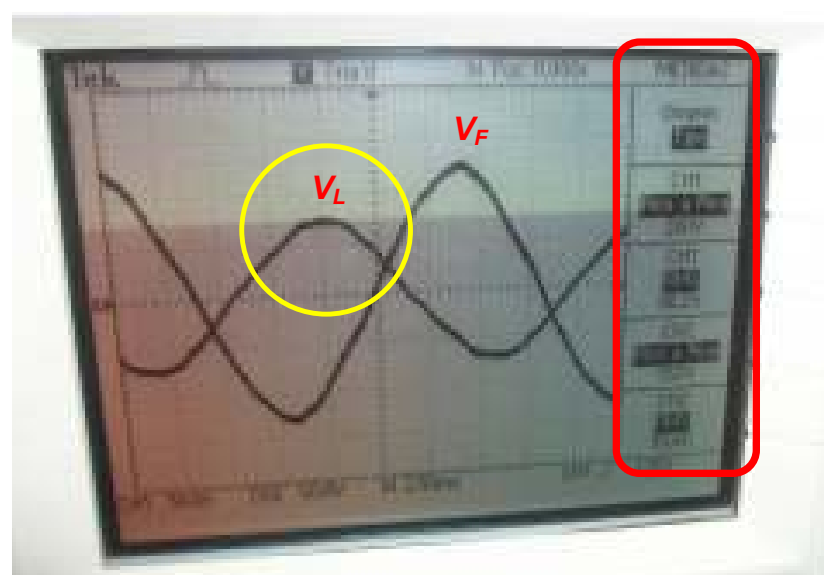

Figura 21 - Formas de Onda de $V_{F}$ e $V_{L}$ do transformador conectado em $\mathrm{Y}-\mathrm{Y}$.

$\mathrm{Na}$ TAB. 3, encontram-se os valores medidos com o osciloscópio.

Tabela 3

Valores Medidos no Osciloscópio

\begin{tabular}{c|c|c|c|c}
\hline & \multicolumn{2}{c|}{ Ligação D-Y } & \multicolumn{2}{c}{ Ligação Y-Y } \\
\cline { 2 - 5 } & $\mathrm{V}_{\mathrm{PP}}[\mathrm{V}]$ & $\mathrm{V}_{\mathrm{RMS}}[\mathrm{V}]$ & $\mathrm{V}_{\mathrm{PP}}[\mathrm{V}]$ & $\mathrm{V}_{\mathrm{RMS}}[\mathrm{V}]$ \\
\hline $\mathrm{CH} 1\left(\mathrm{~V}_{\mathrm{L}}\right)$ & 322 & 107 & 260 & 86,2 \\
\hline $\mathrm{CH} 2\left(\mathrm{~V}_{\mathrm{F}}\right)$ & 118 & 38 & 152 & 50,4 \\
\hline
\end{tabular}

\subsubsection{Discussão dA ANÁlise EXPERIMENTAL II}

Através dos resultados obtidos foi possível comprovar as vantagens da Ligação em Triângulo quando se deseja extinguir as distorções causadas pelo terceiro harmônico. Isso pôde ser observado comparando as formas de onda e analisando os valores de tensão de fase e linha. $O$ terceiro harmônico causou um aumento na amplitude quando o primário foi ligado em Estrela FIG. 21 (detalhe dos círculos amarelos nas Figuras 20 e 21). Comparando os valores da TAB. 3 com os valores reais de tensão $V_{2}=\sqrt{3} V_{F}=220 \mathrm{~V}$, observase que os valores apresentados quando o primário está ligado em Delta estão muito mais próximos dos reais.
Na ligação Y-Y não houve a devida correção de terceiro harmônico, logo ocorreu um "achatamento" da crista da onda e, como consequência, a onda de tensão perdeu em amplitude. Como o valor eficaz é $V_{R u s}=V_{m p} / \sqrt{2}$, também ocorreu uma diminuição significativa no valor de tensão de linha e, portanto um valor eficaz menor. Na ligação D-Y os valores de tensão medidos são maiores, pois com o triângulo no primário houve uma correção do terceiro harmônico, não ocorrendo deformação na forma de onda da tensão de linha, consequentemente o valor de pico a pico e o valor eficaz foram maiores.

\section{CONCLUSÃo}

As distorções harmônicas citadas nesse trabalho são causadas pelo material magnético que constitui o núcleo do equipamento. $O$ transformador tende a atingir níveis de saturação consideráveis em condições normais de operação. Essa condição de operação é necessária para que haja o enlace entre os enrolamentos primário e secundário, entretanto, o transformador fica propício ao aparecimento de harmônicos ímpares, sendo o de terceira ordem o mais prejudicial ao sistema. Os componentes de terceiro harmônico possuem frequências múltiplas de $60 \mathrm{~Hz}$, ou seja, frequência igual a $180 \mathrm{~Hz}$. Além disso, sua amplitude equivale a um terço da amplitude fundamental. Tanto o terceiro harmônico quanto os seus múltiplos caracterizam sistemas trifásicos de sequência zero, isto é, os componentes harmônicos estão em fase nas três linhas do sistema. Essa ideia de propor e demonstrar as vantagens da ligação Triângulo-Estrela partiu da necessidade de se fornecer uma energia de qualidade aos consumidores finais.

Os transformadores sendo máquinas conversoras de energia e utilizadas na maioria dos sistemas de distribuição e conversão de energia precisam trabalhar com um alto rendimento e sem interferências 
eletromagnéticas, pois, essas diminuem o rendimento do equipamento.

A pesquisa experimental realizada teve como objetivo propor uma solução para as interferências eletromagnéticas, causadas pelas distorções harmônicas em transformadores trifásicos. Com relação ao principal objetivo do trabalho, verificou-se que os resultados obtidos estão em conformidade com todo o Referencial Teórico estudado, e, portanto, foi possivel comprovar quão satisfatória é a solução proposta.

\section{AgRADECIMENTOS}

À Deus, a minha família e a todos aqueles que me incentivaram durante o período de estudo.
À professora e Co-Orientadora Arlete pelo ensinamento durante todo $\mathrm{o}$ ano de desenvolvimento desse trabalho.

Ao UniBH e ao PROUNI pela bolsa de estudos concedida e pela oportunidade de me tornar Engenheira.

À PUC Minas, pela oportunidade de realizar ensaios no laboratório de Máquinas Elétricas.

Ao colega de classe Hudson Rodrigues Saldanha pela ajuda no desenvolvimento desse trabalho $\mathrm{e}$ ao Instrutor do Laboratório de Eletrônica do UniBH Marcos Elias Gomes Doerl, pela atenção concedida durante os ensaios práticos.

OLIVEIRA, J. C. Transformadores: Teoria e Ensaios. São Paulo/ SP: Editora Edgard Blucher. 1984. 174p.

SILVA JÚNIOR, P. A. Bancada para Ensaios de Materiais Ferromagnéticos em chapa única sob campos rotacionais. Florianópolis/ SC. UFSC. 2007. Disponível em:

https://wiki.ifsc.edu.br/mediawiki/images/3/3b/Tese Pe droArmando.pdf Acesso em: 02 mai. 2012.

STEVENSON, W. Elementos de Análise de Sistemas de Potência. São Paulo/ SP: Editora McGraw Hill. 1986. 452p.

TORO, V. D. Fundamentos de Máquinas Elétricas. Rio de Janeiro/ RJ: Editora LTC. 1999. 541p. 